Gerd Kaminski, Oskar Weggel (Hrsg.)

China und das Völkerrecht

Mitteilungen des Instituts für Asienkunde, Nr. 126, Hamburg, 1982, XII, 282 S., DM 28,-

Im Anschluß an eine Tagung im November 1977 in Hamburg ${ }^{1}$ ist ein Sammelband erschienen, der zum Teil die Referate und weitere Beiträge, insbesondere von Autoren aus der Volksrepublik China, enthält.

Neben einem geschichtlichen Abriß über die ersten Kontakte des chinesischen kaiserlichen Staates mit dem westlichen Völkerrecht (leider ohne Darstellung des traditionellen Systems der internationalen Beziehungen im Fernen Osten) und Beschreibungen zur Haltung und Mitarbeit der Volksrepublik China in internationalen Organisationen bietet der Band auch eine Skizze über chinesische Positionen zum Kriegsvölkerrecht von Robert Heuser. Die Ausführungen in dem Beitrag von Li Haopei zum internationalen Privatrecht der Volksrepublik China sind zum Teil durch neue Literatur und Gesetzgebung überholt. ${ }^{2}$ Ein Referat der Tagung, "Das Territorium im chinesischen Völkerrecht", wird als separate Veröffentlichung von Michael Strupp erscheinen.

Wolfgang Kessler

Jorge Carpizo

\title{
La Constitución Mexicana de 1917
}

4. Aufl., Universidad Nacional Autónoma de México, 1980, 317 S., US-\$ 7,50.

Den programmatischen Charakter der Verfassung über die aktuelle Geltung als derzeitige Fundamentalnorm hinaus definiert $\mathrm{C}$. plastisch als Begegnung des Menschen mit seiner Epoche, als Dialog des heutigen Bürgers mit der Geschichte in allen ihren früheren Auseinandersetzungen und als die Aufnahme des Gesprächs mit künftigen Generationen. Und dennoch beinhaltet der Verfassungstext einen Monolog, so wie er von den Verfassungsgebern in einem bestimmten historischen Prozeß 1917 einseitig auf gewachsene und auf antizipierte Fragen hin festgeschrieben worden ist. Ein verhältnismäßig junger Staat mit außerordentlich unterschiedlichen ethnischen und sozialen Basisdaten wird und muß sich um seines eigenen Nationwerdens willen die Geschichte und auch die seiner Verfassung immer wieder vergegenwärtigen. Daß sich heute die Einwohner Mexikos tatsächlich als Mexikaner fühlen, ist nicht selbstverständlich, sondern Ergebnis eines

1 Vgl. Kunig, Arbeitstagung "Das chinesische Völkerrechtssystem", VRU 11 (1978), S. $137 \mathrm{f}$.

2 Z. B. Yao Zhuang/Ren Jisheng, Guoji sifa jichu, Peking, Zhongguo shehui kexue chubanshe, 1981; Bestimmungen zum internationalen Privatrecht in der neuen ZPO-Volksrepublik China vom 8. März 1982 - eine, leider of t nicht verläßliche, Ubersetzung dieser ZPO findet sich in: China aktuell 1982 (März), 135 ff. 
mühseligen Prozesses. Es darf daher den Leser nicht verwundern, daß auch diese Arbeit der Entstehungsgeschichte der Verfassung von 1917 einen für europäische Verhältnisse breiten Raum einräumt. Hinzu kommt, daß der Legitimationsgrad der Verfassungsgebung 1917 nicht überragend groß gewesen und erst im Nachhinein mit den späteren Wahlen nach Maßgabe dieser Verfassung nachgeholt worden ist.

Sowohl in der Darstellung der Geschichte der Grundsatzentscheidungen als auch der ihrer inhaltlichen Beurteilung werden Schwerpunkte gesetzt: Es geht um das Unterrichtswesen und damit in diesem katholischen Land eng zusammenhängend um die religiöse Frage und um das institutionelle Abdrängen der Kirche aus dem öffentlichen Leben. Es geht um die großen sozialen Fragen - erstmals in einer Verfassung enthalten - und die Landreform.

Die historisch keineswegs selbstverständliche Entscheidung für den Föderalismus wird als eine zwangsläufige Fundamentalentscheidung dargestellt. Den Kompensationscharakter der entsprechenden verfassungsrechtlichen Dezisionen kann auch C. nicht ganz verbergen. Die Entstehungsgeschichte der mexikanischen Einzelstaaten nach Maßgabe der spanischen Verfassung von Cadiz, die 1812 für jede Provinz eine Deputation vorsah und auch für Nueva España zur Bildung von sechs Deputationen (in Ciudad de México, Monterrey, Guadalajara, Durango, Guatemala und Mérida) führte, zeigt zunächst nur, daß bestehende spanische Verwaltungsregionen in den Händen der dortigen kreolischen Oberschicht für eigene Zwecke benutzt wurden. Ein irgendwie an die Region gebundenes ethnisches, historisches oder auch nur ökonomisch-geographisches Substrat als Basis für einen hieraus definierbaren Staat wird nicht erkennbar, es gibt es auch kaum: Aus der vorkolonialen Zeit sind nur die Gebiete der Indios im Tal von Tlaxcala und Puebla, den alten Verbündeten der Spanier gegen die Azteken, fast unverändert in die Kolonialverwaltung übernommen worden. Dort jedoch, wo der kolonialen Verwaltungsstruktur ein eigenständiger geopolitischer Raum mit separaten wirtschaftlichen Notwendigkeiten entspricht, ist eine reale Staatsbildung indiziert. Guatemala, im Generalkapitanat bereits vom Rest des Vizekönigreichs gesondert, erlangte 1824 seine Selbständigkeit von Mexiko. Yucatán, ebenfalls ohne ausreichende Verbindung zu Zentralmexiko, seewärts gerichtet mit starken Handelsbeziehungen zu den USA, schien anfangs den gleichen Weg wie Guatemala zu gehen. Die auf sich selbst angewiesenen Bewohner Yucatáns hatten jedenfalls keine substantiellen Bindungen zu den anderen Provinzen, von denen schließlich 1823 insgesamt 23 mit eigenen Deputationen existierten. In den Wirren des Kaiserreichs Iturbides bedeutete die Selbstverwaltung in den Provinzen mangels Zentralgewalt, daß sie wie Staaten agieren mußten. Mehrere Provinzen erklärten sich 1823 für frei und souverän. In dieser Situation war die Begründung eines Bundesstaates als $\mathrm{Zu}$ sammenschluß der Stücke des zerbröckelnden Staatsverbandes eine politische Not- und Mindestlösung. Die Forderung nach einem Zentralstaat konnte sich angesichts der Rivalitäten der in den Peripherien Herrschenden nicht durchsetzen. Es wäre demnach sicherlich falsch, die Geburt des Bundesstaates in Mexiko mit dem Zusammenschluß der eigenständigen deutschen Einzelstaaten im vorigen Jahrhundert zu vergleichen. "Souverän" in den Erklärungen der mexikanischen Provinzen wurde damals daher durchaus zu- 
treffend eher mit "autonom", nämlich keiner anderen Gewalt als der eigenen Regierung unterliegend, verstanden (C., S. 243 ff.). Die staatsrechtliche Qualifikation als Einzelstaaten erhielten die Provinzen erst mit den Verfassungsgesetzen v. 31. Jan. und 4. Okt. 1824. Mag mit einer Unterbrechung Mitte des vorigen Jahrhunderts der Föderalismus in Mexiko verfassungsrechtlich jeweils abgesichert gewesen sein, seine Lebenskraft hängt nicht von den Kompetenzverteilungen im Verhältnis zwischen Gliedstaaten und Bund $\mathrm{ab}$, deren systematische Unterteilung C. sieben Seiten widmet, sondern von der Möglichkeit, divergierende politisch-ökonomische Unterschiede herauszubilden und im Gesamtstaat zum Ausgleich bringen zu können. Mit der Anbindung Yucatáns nach dem Separationsversuch von 1840 mittels einer Eisenbahnlinie an Zentralmexiko und der Aufteilung der Halbinsel in zwei Staaten - Yucatán (mit der alten Hauptstadt Mérida) und Campeche, dem zum mexikanischen Markt ausgerichteten westlichen Teil der Halbinsel - waren wirkliche Schwierigkeiten mit zentrifugalen Kräften seit langem nicht mehr vorhanden. Guatemala und Yucatán bilden die Beispiele eines gelungenen und eines erfolglosen Separatismus. Der Abfall von Texas vom mexikanischen Gesamtstaat fällt aus dem hier besprochenen Rahmen, da jener Ergebnis einer protestantisch-nordamerikanischen Bevölkerungsinfiltration war.

Mit der Dauerherrschaft des regierenden Partido Revolucionario Institucional auf allen Ebenen des staatlichen Lebens gibt es auch heute keinen Föderalismus, sondern nur einen - wie C., S. 250, abschließend ohne notwendig werdenden besonderen Beleg ${ }^{1}$ meint - gemäßigten und bei anderer Gelegenheit verschärften Zentralismus.

Auffällig ist die verhaltene Distanz des Autors zu der augenfälligen Diskrepanz zwischen der realen und der geschriebenen Verfassung des Landes. Auf erstere wird nur gelegentlich verwiesen, wenn etwa auf die tatsächliche Machtfülle des Präsidenten und deren Grenzen eingegangen wird: Erwähnt wird die Uberschreitung des Kreditrahmens im Haushalt 1976 um 48,4\% einerseits und die Presse andererseits (S. 304), die angesichts der geringen Zahl der Leser allenfalls eine bloße Belästigung der Regierenden ist. Carpizos Darstellung ist nach allem die der idealen Verfassung im Spiegel ihres geschriebenen Textes.

Gerhard Scheffler

1 Der mexikanische Leser bedarf eines solchen nicht, er hat ihn tagtäglich vor Augen; für den deutschen Leser sei auf Wolfgang Enders, Der mexikanische Bundesstaat, Frankfurt a. M.-Bern-Las Vegas, 1977, verwiesen. 